Original Article

\title{
Concomitant Mitral Regurgitation in Patients Undergoing Surgical Aortic Valve Replacement for Aortic Stenosis: A Systematic Review
}

\author{
Francis P. Cheung, Cheng He, Philippa R. Eaton, Jim Dimitriou, and Andrew E. Newcomb
}

\begin{abstract}
Background: Concomitant mitral regurgitation (MR) is frequently seen in patients undergoing surgical aortic valve replacement (AVR) for severe aortic stenosis (AS). When the severity of MR is moderate or less, the decision to undertake simultaneous mitral valve intervention can be challenging.

Methods: A systematic search of Medline, PubMed (NCBI), Embase and Cochrane Library was conducted to qualitatively assess the current evidence for concomitant mitral valve intervention for MR in patients with AS undergoing AVR. The primary outcome for this systematic review was the postoperative change in the severity of MR and other outcomes of interest included factors that predict improvement or persistence of MR and long-term impacts of residual MR.

Results: A total of 17 studies were included. The percentage of patients demonstrating improvement in MR severity following AVR ranged from $17.2 \%$ to $72 \%$; the studies that exclusively included patients with moderate functional MR and reported longer term echocardiographic follow-up of greater than 12 months demonstrated an improvement in MR severity of $45 \%$ to $72 \%$.

Conclusion: This systematic review demonstrates that a proportion of patients can exhibit an improvement in MR following isolated surgical AVR, but whether this confers any long-term morbidity and mortality benefit remains unclear.
\end{abstract}

Keywords: mitral regurgitation, aortic stenosis, aortic valve replacement

\section{Introduction}

Mitral regurgitation (MR) is frequently observed concomitantly in patients with severe aortic stenosis (AS)

Department of Cardiothoracic Surgery, St Vincent's Hospital Melbourne, Fitzroy, VIC, Australia

Received: July 26, 2021; Accepted: December 10, 2021

Corresponding author: Francis Park-Yun Cheung. Department of Cardiothoracic Surgery, St Vincent's Hospital Melbourne, 41 Victoria Parade, Fitzroy, VIC 3065, Australia

Email: francispcheung@outlook.com

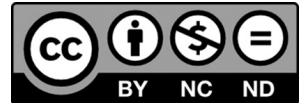

This work is licensed under a Creative Commons Attribution-NonCommercialNoDerivatives International License.

(C)2022 The Editorial Committee of Annals of Thoracic and Cardiovascular Surgery who undergo aortic valve replacement (AVR), with a reported incidence of up to $75 \% .^{1,2)}$ The aorto-mitral apparatus exists in a dynamic functional balance and persistent left ventricular wall stress from severe AS not only leads to concentric hypertrophy but also increases trans-mitral pressure gradients during systole. ${ }^{1,3,4)}$ This can lead to secondary functional MR, particularly if the left ventricle (LV) or mitral annulus is dilated. Alternatively, primary MR may be exacerbated by the same process. ${ }^{4,5)}$ In the setting of severe functional or primary MR, concomitant mitral valve surgery is reasonably indicated in accordance with the current guidelines. ${ }^{6-8)}$ However, when the degree of MR is moderate or less, the clinical decision to intervene is often held in equipoise. ${ }^{9)}$

The severity of functional MR can be expected to improve following an AVR with myocardial remodeling 
Cheung FP, et al.

and subsequent enhancement of overall haemodynamics. ${ }^{1)}$ With this anticipated improvement and the increasing morbidity and mortality risk of a double valve intervention, expectant management of concomitant MR is justifiable when the severity is moderate or less. ${ }^{9)}$ However, the degree of change in MR following AVR varies considerably and the proportion of patients exhibiting improvement is inconsistent, ranging from $23 \%$ to $82 \%{ }^{10-12)}$ Conflicting reports have also demonstrated a proportion of patients who experience unchanged or worsening severity of MR. ${ }^{13)}$ In addition, poorer longterm outcomes in patients left with persistent MR following AVR have been reported in some studies. ${ }^{1,14,15)}$ Specifically, during follow-up, some patients exhibiting persistent MR appear to experience worsening overall survival and more frequent heart failure-related hospital admissions, which may provide sound rationale for more aggressive double valve intervention in carefully selected patients. ${ }^{14,15)}$

The natural history and prognostic impact of persistent functional MR following AVR remains unclear. In the absence of robust evidence to guide management of concomitant moderate MR, consideration of patients' life expectancy influences the Heart Team's decision to recommend double valve surgery or not, but echocardiographic predictors of MR improvement following AVR also need to be more clearly defined. ${ }^{1,2)}$ This systematic review aims to qualitatively assess the current evidence for mitral valve intervention when patients present with severe AS and concomitant functional MR.

\section{Methods}

\section{Search strategy}

A systematic search of published literature was conducted in accordance with the Preferred Reporting Items for Systematic Reviews and Meta-Analyses (PRISMA). The study team developed a concept table and subsequent search strategy to identify articles reporting outcomes on concomitant MR in patients with AS undergoing surgical AVR. Two main search concepts were developed: MR and AVR. Exploded medical subject heading terms were combined with keyword searches using the Boolean operator 'OR' for each concept, with each concept then combined using 'AND' (see Appendix 1). Spelling variations were accounted for using ' $*$ ' in the search to represent wildcard characters. No search limits were applied. The five databases searched included Medline (Ovid: 1946-present), PubMed (NCBI), Embase (Ovid: 1966-present) and Cochrane Library. The final search date was 1 July 2020. Reference lists of included articles were also searched. The full search strategy used is summarised in Fig. 1 and Appendix 1.

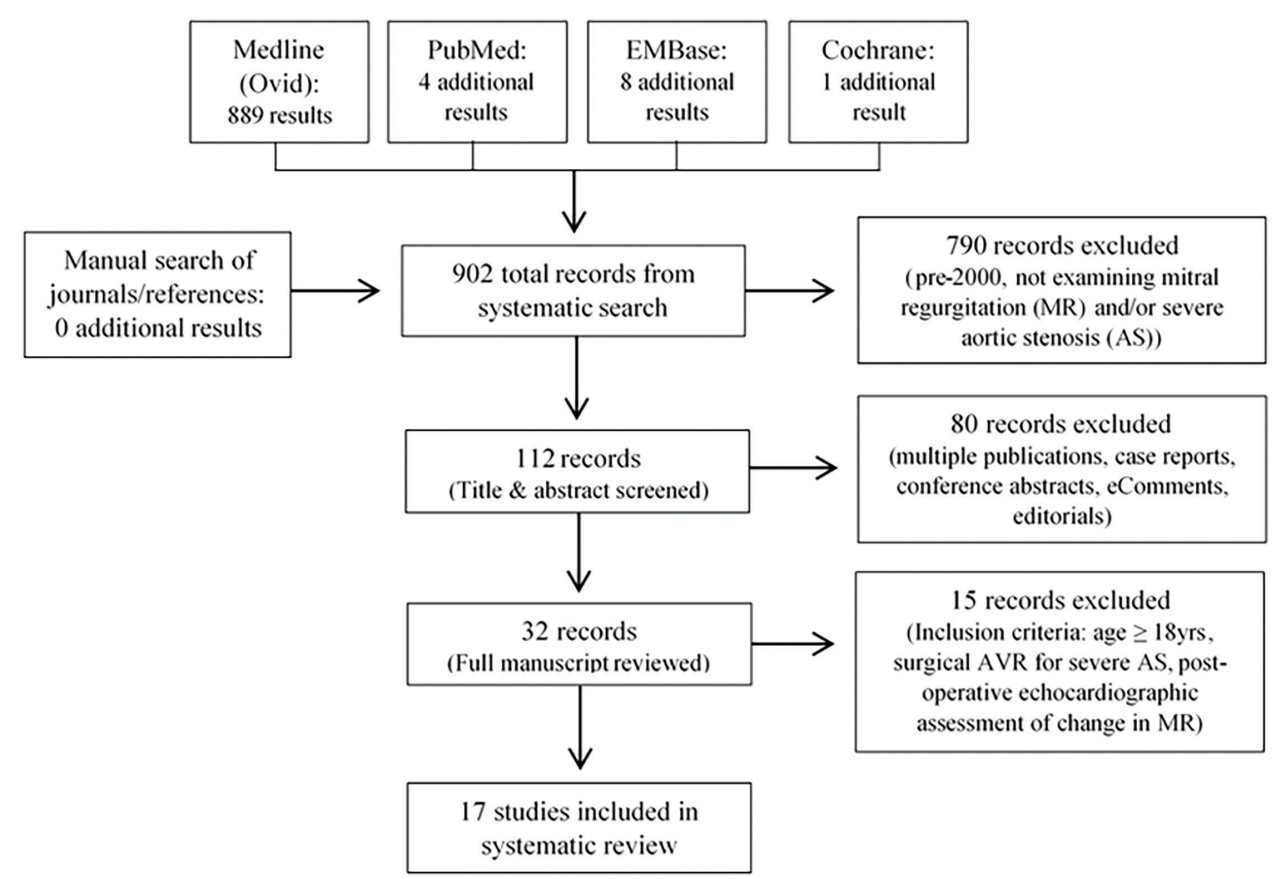

Fig. 1 Study selection process. MR: mitral regurgitation; AS: aortic stenosis; AVR: aortic valve replacement 


\section{Identification of studies and risk of bias assessment}

Eligibility criteria for this literature review were pre-determined (Appendix 2), and two authors independently assessed the study titles and abstracts for inclusion. Any differences in title selection between them were discussed and resolved with a third assessor independently reconciling any differences in selection. Full-text versions of potentially eligible studies were further reviewed by two independent study authors against the inclusion or exclusion criteria. All relevant prospective and retrospective studies were identified. To date, no randomized controlled trials (RCTs) investigating outcomes of concomitant MR following surgical AVR have been published. Any case reports or small series from single centers ( $\leq 5$ patients) were excluded to minimize selection, reporting and publication bias.

Studies considered eligible for inclusion reported on adult patients aged over 18 years undergoing surgical AVR with concomitant MR and measuring postoperative change in severity of MR as a primary outcome measure. Any studies only utilizing intraoperative transoesophageal echocardiographic assessment of MR were excluded. Studies reporting surgical outcomes that included patients undergoing mitral valve procedures were also excluded. In order for this literature review to reflect outcomes for patients undergoing modern surgical, anesthetic and diagnostic imaging techniques, only articles published in the last 20 years since 2000 were included.
Two study authors independently assessed the risk of bias using the Newcastle Ottawa Scale for cohort studies and a third author resolved any discrepancies (Table 1). Studies were assigned a score based on selection, comparability and outcomes using the published criteria.

\section{Data extraction and outcomes}

One author extracted information from the studies using a standardized spreadsheet. Data extracted included study period, study design, aim, patient demographics, cohort number, primary operation, etiology of MR, severity of MR and degree of improvement postoperatively, echocardiographic modality and follow-up period. The heterogeneous nature of the methodology, data collection and reported outcomes in the included studies did not allow for meta-analysis to be conducted in this systematic review.

\section{Results}

\section{Search results}

The final 17 studies included in this systematic review (Table 2) included 14 retrospective and 3 prospective cohort studies. All studies were published within the last 20 years since 2000 and the study periods ranged from 1990 to 2017. A total of 2281 patients were included from all studies, with median age ranging from 53 years

Table 1 Newcaslte Ottawa Risk of Bias Assessment

\begin{tabular}{|c|c|c|c|c|}
\hline \multicolumn{5}{|c|}{ Newcastle Ottawa Risk of Bias Assessment for Cohort Studies } \\
\hline Study & $\begin{array}{c}\text { Selection } \\
(\text { maximum }=4)\end{array}$ & $\begin{array}{c}\text { Comparability } \\
(\text { maximum }=2)\end{array}$ & $\begin{array}{c}\text { Outcome } \\
(\text { maximum }=3)\end{array}$ & $\begin{array}{l}\text { Overall quality } \\
(\text { maximum }=9)\end{array}$ \\
\hline Brasch et al. ${ }^{19)}$ & $* * * *$ & $*$ & $* *$ & 7 \\
\hline Christenson et al. ${ }^{16)}$ & $* * * *$ & $*$ & $* *$ & 7 \\
\hline Absil et al. ${ }^{17)}$ & $* * * *$ & $*$ & $* * *$ & 8 \\
\hline Goland et al. ${ }^{18)}$ & $* * * *$ & $*$ & $* * *$ & 8 \\
\hline Tassan-Mangina et al. ${ }^{20)}$ & $* *$ & $*$ & $* *$ & 5 \\
\hline Moazami et al. ${ }^{1)}$ & $* * * *$ & $*$ & $* * *$ & 8 \\
\hline Vanden Eynden et al. ${ }^{11)}$ & $* * * *$ & $*$ & $* * *$ & 8 \\
\hline Caballero-Borrego et al. ${ }^{22)}$ & $* * *$ & $*$ & $* * *$ & 7 \\
\hline Unger et al. ${ }^{10)}$ & $* * *$ & $*$ & $* *$ & 6 \\
\hline Wan et al. ${ }^{2)}$ & $* * * *$ & $*$ & $* * *$ & 8 \\
\hline Takeda et al. ${ }^{15)}$ & $* * * *$ & $*$ & $* * *$ & 8 \\
\hline Joo et al. ${ }^{14)}$ & $* * * *$ & $*$ & $* * *$ & 8 \\
\hline Aljadayel et al. ${ }^{21)}$ & $* * * *$ & $*$ & $* *$ & 7 \\
\hline Khosravi et al. ${ }^{28)}$ & $* * *$ & $*$ & $* *$ & 6 \\
\hline Sehovic et al. ${ }^{29)}$ & $* * *$ & $*$ & $* *$ & 6 \\
\hline Schubert et al. ${ }^{13)}$ & $* * * *$ & $*$ & $* *$ & 7 \\
\hline Wang et al. ${ }^{9)}$ & $* * * *$ & $*$ & $* *$ & 7 \\
\hline
\end{tabular}


Cheung FP, et al.

Table 2 Study characteristics

\begin{tabular}{|c|c|c|c|c|c|}
\hline Authors & Pub year & Study type & Period & Primary outcome & $\mathrm{n}$ \\
\hline Brasch et al. ${ }^{19)}$ & 2000 & Retrospective cohort & 1995-1999 & Assess change in MR severity & 27 \\
\hline Christenson et al. ${ }^{16)}$ & 2000 & Retrospective cohort & 1990-1999 & Assess change in MR severity & 60 \\
\hline Absil et al. ${ }^{17)}$ & 2003 & $\begin{array}{l}\text { Retrospective case- } \\
\text { matched }\end{array}$ & $1992-2000$ & Assess change in MR severity & 116 \\
\hline Goland et al. ${ }^{18)}$ & 2003 & Retrospective cohort & 1996-1999 & Determine survival outcomes & 30 \\
\hline Tassan-Mangina et al. ${ }^{20)}$ & 2003 & Prospective cohort & - & Assess change in MR severity & 30 \\
\hline Moazami et al.1) & 2004 & Retrospective cohort & 1991-2001 & Assess change in MR severity & 107 \\
\hline $\begin{array}{l}\text { Vanden Eynden } \\
\text { et al. }{ }^{11)}\end{array}$ & 2007 & Retrospective cohort & 1994-1996 & Assess change in MR severity & 80 \\
\hline $\begin{array}{l}\text { Caballero-Borrego } \\
\text { et al. }{ }^{22)}\end{array}$ & 2008 & Retrospective cohort & 1996-2007 & Assess change in MR severity & 577 \\
\hline Unger et al. ${ }^{10)}$ & 2008 & Prospective cohort & - & $\begin{array}{l}\text { Analysis of pre-operative } \\
\text { predictors of MR change }\end{array}$ & 52 \\
\hline Wan et al. ${ }^{2)}$ & 2009 & Retrospective cohort & 1993-2006 & Assess change in MR severity & 190 \\
\hline Takeda et al. ${ }^{15)}$ & 2010 & Retrospective cohort & 1993-2007 & $\begin{array}{l}\text { Assess change in MR severity } \\
\text { and long term outcomes }\end{array}$ & 193 \\
\hline Joo et al. ${ }^{14)}$ & 2011 & Retrospective cohort & 2000-2009 & Assess change in MR severity & 118 \\
\hline Aljadayel et al. ${ }^{21)}$ & 2015 & Retrospective cohort & 2005-2012 & Assess change in MR severity & 149 \\
\hline Khosravi et al. ${ }^{28)}$ & 2015 & Prospective cohort & 2011-2012 & Assess change in MR severity & 85 \\
\hline Sehovic et al. ${ }^{29)}$ & 2015 & Retrospective cohort & 2011-2013 & Assess change in MR severity & 45 \\
\hline Schubert et al. ${ }^{13)}$ & 2016 & Retrospective cohort & 2004-2013 & Assess change in MR severity & 423 \\
\hline Wang et al. ${ }^{9)}$ & 2019 & Retrospective cohort & 2013-2017 & Assess change in MR severity & 49 \\
\hline
\end{tabular}

MR: mitral regurgitation

to 77 years in the studies and a total 1791 patients included exhibited concomitant MR at the time of AVR.

\section{Surgical cohort and etiology of MR}

The surgical cohorts of the final 17 studies included 14 studies with patients undergoing isolated AVR, 2 studies with a mixed AVR or AVR plus coronary artery bypass graft (CABG) cohort and 1 study with a heterogeneous cohort of aortic valve or root replacement with or without other minor procedures. ${ }^{15)}$ The clinical indication for AVR was outlined in all 17 studies, with 11 studies including only patients with severe AS and 6 studies including a mixed cohort of AS and/ or aortic regurgitation. The etiology of MR was reported in 15 studies, with 10 studies examining only patients with functional MR and 5 studies examining a more heterogeneous cohort of both primary and secondary MR. The majority of patients $(68.4 \%)$ in all studies had functional MR.

\section{Degree and measurement of MR severity}

The preoperative severity of MR was outlined in 12 studies. Vanden Eynden et al., Wan et al. and Wang et al. were the 3 studies that exclusively included moderate or moderate to severe MR only in their cohorts, whilst the remaining studies included a range from trace to severe
MR. The echocardiographic modalities used to evaluate MR severity did vary between studies. Transthoracic echocardiography (TTE) was used to assess pre- and postoperative MR in 16 studies, and combined TTE and transoesophageal echocardiography (TOE) was used in 1 study. The specific echocardiographic parameters used to qualitatively and/or quantitatively assess MR severity were outlined in 13 studies. The most commonly reported method of assessment was using proximal isovelocity surface area (PISA) and/or effective regurgitant orifice area (EROA) in 7 studies, whilst 6 studies utilized MR jet area and/or vena contracta width.

\section{Postoperative change in severity of MR}

All studies performed pre- and postoperative echocardiographic assessment of MR severity (Table 3); however, a range of follow-up time periods was reported for postoperative echocardiograms. A total of 14 studies reported the follow-up timeframe for postoperative TTE or TOE: 8 studies conducted echocardiograms within 1 year following surgery and the remaining 6 studies ranged from 12 months up to 36 months following surgery. A total of 14 studies reported the degree of change in MR severity following AVR. Within these studies, the percentage of patients demonstrating improvement in MR severity ranged widely from $17.2 \%$ to $72 \%$, but the large 
majority, 15 studies, reported an improvement seen in $43.7 \%$ to $72 \%$ of patients. ${ }^{16)}$ The percentage of patients exhibiting worsening MR following AVR ranged from $0 \%$ to $27.1 \%$ and no change in MR severity was seen in $22 \%$ to $56.2 \%$ of patients of the included studies. ${ }^{111)}$ Of note, the 4 studies that exclusively included patients with functional MR and reported longer term echocardiographic follow-up of greater than 12 months demonstrated an improvement in MR severity ranging from $45 \%$ to $72 \% .1,14,17,18)$

\section{Predictors of change in MR severity}

The clinical and echocardiographic factors predicting change in MR severity were analyzed in 13 studies. In 7 studies, significant preoperative factors were found to predict improvement in MR, in particular increased LV mass or hypertrophy and LV end-diastolic diameter or volume. ${ }^{10,19,20)}$ Other factors included the presence of coronary artery disease, lower left ventricular ejection fraction (LVEF) and increased left atrial size.,21) Independent factors associated with persistent MR post AVR were analyzed in 3 studies and 2 of these identified significant comorbid factors, which included elevated right ventricular systolic pressure or pulmonary hypertension and comorbid diabetes mellitus. ${ }^{14,21,22)}$

\section{Long-term impact of preoperative and postoperative MR}

The impact of preoperative MR severity on long-term morbidity and overall survival was reported in 7 studies, with 4 studies demonstrating significant impact. ${ }^{1,11,14,15)}$ Takeda et al. found that patients with mild to moderate preoperative MR or those with an LVEF $<50 \%$ were at an increased risk of heart failure-related readmissions than those with trace to mild MR or an LVEF $\geq 50 \% .{ }^{15}$ In addition, Moazami et al. reported that short- to mediumterm survival is worse in patients with greater degrees of preoperative MR. ${ }^{1)}$ Overall survival at 1 year and 3 years in patients with moderate or severe preoperative MR was $97 \%$ and $78 \%$, respectively, compared with $99 \%$ in those patients with trivial or mild preoperative MR $(\mathrm{p}=$ 0.038). ${ }^{1)}$ However, in the studies that did not include patients with severe grades of MR, there was no significant impact of preoperative MR severity on the overall survival. ${ }^{13,15,17)}$

The influence of MR improvement or persistence on survival outcomes varied between the reported studies. Joo et al. reported that patients with persistent postoperative MR predicted worse long-term survival compared with patients who exhibited no residual MR, with 10 -year survival rates of $77.8 \%$ and $93.1 \%$, respectively $\left.(p=0.036) .{ }^{14}\right)$ Vanden Eynden et al. also found that patients with MR improvement after isolated AVR held significantly better 10-year survival than patients who had no change or worsening MR severity. ${ }^{11)}$ Schubert et al. identified a trend towards improved survival in patients with MR improvement with a 5-year survival of $73.5 \%$ compared with $65.4 \%$ in patients whose MR remained the same, but was not statistically significant $\left.(\mathrm{p}=0.06){ }^{13}\right)$ Three of the reported studies, however, reported no significant difference in long-term survival when comparing patients with improved MR to those with residual MR following an AVR.,1,217)

\section{Discussion}

Historically, the aortic and mitral valves have been studied as independent structures with isolated function, but increasingly we understand the aortomitral apparatus to exist in dynamic synchrony.5) The aortic and mitral valves are no longer considered two separate entities but rather function in a dynamic equilibrium where each valve imparts geometric changes on the other throughout the cardiac cycle. ${ }^{5)} \mathrm{AS}$ is the most common cardiac valve pathology and has been linked with the disturbance of this aortomitral synchrony, potentially causing or contributing to concomitant mitral valve regurgitation. ${ }^{4,5)}$ Whilst primary MR stems directly from structural pathology, secondary functional MR develops despite a structurally sound mitral valve apparatus, when abnormal left ventricular geometry (or in some cases left atrial) disrupts the delicate balance between closing and tethering forces on the mitral valve., ${ }^{5,23)}$ This is seen often in the context of dilated cardiomyopathy or ischaemic heart disease or even in severe left atrial dilatation. ${ }^{24)}$

In the setting of severe AS, functional MR can often coexist. ${ }^{9)}$ The chronic increase in transaortic valve gradient and intraventricular pressure coupled with subsequent LV remodeling is thought to provoke this secondary MR. The reduction in aortic valve gradient following an AVR has the potential to improve any concomitant MR as a result of decreased LV pressure, altered mitral annulus size and reverse LV remodeling in the long term. ${ }^{3,4)}$ However, the current largely retrospective evidence base remains inconsistent and considerable variability exists in the percentage of patients who demonstrate postoperative improvement. ${ }^{13)}$ Whilst the majority of studies in this review identified MR 


\begin{tabular}{|c|c|c|c|c|c|c|c|c|c|c|}
\hline \multirow{2}{*}{ Study } & \multicolumn{2}{|c|}{ Primary operation (n) } & \multicolumn{2}{|c|}{ Preoperative MR aetiology (n) } & \multicolumn{3}{|c|}{ Postoperative change in MR severity \% (n) } & \multirow{2}{*}{$\begin{array}{c}\begin{array}{c}\text { Echocardiographic } \\
\text { assessment (TTE/TOE) }\end{array} \\
\text { (Post-op timeframe) }\end{array}$} & \multirow[t]{2}{*}{$\begin{array}{l}\text { Predictors of postoperative } \\
\text { MR change }\end{array}$} & \multirow{2}{*}{ p-value } \\
\hline & AVR & $\begin{array}{l}\mathrm{AVR}+ \\
\mathrm{CABG}\end{array}$ & Functional & Other & Improved & No change & Worse & & & \\
\hline Brasch et al. ${ }^{19)}$ & 27 & - & $23(85 \%)$ & Leaflet restriction: 4 & $44 \%(12)$ & $52 \%(14)$ & $4 \%(1)$ & TTE (2.2 months) & Left ventricular mass & $<0.05$ \\
\hline Christenson et al. ${ }^{16)}$ & 60 & - & $58(100 \%)$ & - & $55.2 \%(32)$ & $17.2 \%(10)$ & $0 \%$ & TTE (2.7 months) & - & - \\
\hline Absil et al. ${ }^{17)}$ & 116 & - & $116(100 \%)$ & - & $31 \%(18)$ & $69 \%(40)$ & $0 \%$ & $\begin{array}{l}\text { TTE (12 months) } \\
\text { MR jet area }\end{array}$ & - & - \\
\hline Goland et al. ${ }^{18)}$ & 30 & - & $30(100 \%)$ & - & $40 \%(12)$ & $60 \%(18)$ & $0 \%$ & $\begin{array}{l}\text { TTE (13 months) } \\
\text { MR jet area }\end{array}$ & - & - \\
\hline $\begin{array}{l}\text { Tassan-Mangina } \\
\text { et al. }{ }^{20)}\end{array}$ & 27 & 3 & - & - & - & - & - & $\begin{array}{c}\text { TTE + TOE (19 days) } \\
\text { MR jet area }\end{array}$ & $\begin{array}{l}\text { Left ventricular mass } \\
\quad \text { Peak tricuspid } \\
\text { regurgitation velocity }\end{array}$ & $\begin{array}{c}0.009 \\
0.02\end{array}$ \\
\hline Moazami et al. ${ }^{1)}$ & 107 & - & $\begin{array}{c}107 \\
(100 \%)\end{array}$ & - & $28 \%(30)$ & $44.9 \%(48)$ & $27.1 \%(29)$ & TTE (26.9 months) & $\begin{array}{c}\text { Preoperative myocardial } \\
\text { infarct }\end{array}$ & 0.014 \\
\hline $\begin{array}{l}\text { Vanden Eynden } \\
\text { et al. }{ }^{11)}\end{array}$ & 80 & - & $14(20 \%)$ & $\begin{array}{l}\text { Organic: } 38 \\
\text { Ischaemic: } 26\end{array}$ & $56.2 \%(9)^{\ddagger}$ & $43.8 \%(7)^{\ddagger}$ & $0 \%$ & $\begin{array}{l}\text { TTE (12 months) } \\
\text { MR jet area }\end{array}$ & $\dagger$ & - \\
\hline $\begin{array}{l}\text { Caballero-Borrego } \\
\text { et al. }{ }^{22)}\end{array}$ & 577 & - & $\begin{array}{c}153 \\
(100 \%)\end{array}$ & - & $22.8 \%(35)$ & $72 \%(110)$ & $5.2 \%(8)$ & $\begin{array}{c}\mathrm{TTE}^{\neq} \\
\text {MR jet area }\end{array}$ & Coronary artery disease & 0.038 \\
\hline Unger et al. ${ }^{10)}$ & 33 & 19 & $28(54 \%)$ & Organic: 24 & - & - & - & $\begin{array}{l}\text { TTE (8.3 days) } \\
\text { PISA/EROA }\end{array}$ & $\begin{array}{l}\text { Left ventricle end-diastolic } \\
\text { volume }\end{array}$ & $<0.001$ \\
\hline Wan et al. ${ }^{2)}$ & 190 & - & $\begin{array}{c}190 \\
(100 \%)\end{array}$ & - & $72 \%(114)$ & $26 \%(42)$ & $2 \%(3)$ & $\begin{array}{l}\mathrm{TTE}^{\ddagger} \\
P I S A\end{array}$ & $\begin{array}{l}\text { Less tricuspid regurgitation } \\
\text { Lower left ventricular } \\
\text { ejection fraction }\end{array}$ & $\begin{array}{l}0.02 \\
0.01\end{array}$ \\
\hline Takeda et al. ${ }^{15)}$ & 193 & - & $24(41 \%)$ & Organic: 35 & $60.3 \%(35)$ & $36.2 \%(21)$ & $3.4 \%(2)$ & $\begin{array}{l}\text { TTE (12-36 months) } \\
\text { MR jet distance }\end{array}$ & $\dagger$ & - \\
\hline Joo et al. ${ }^{14)}$ & 118 & - & $\begin{array}{c}118 \\
(100 \%)\end{array}$ & - & $72 \%(81)$ & $25 \%(28)$ & $3 \%(4)$ & $\begin{array}{c}\text { TTE (56.7 months) } \\
\text { PISA }\end{array}$ & $\dagger$ & - \\
\hline Aljadayel et al. ${ }^{21)}$ & 149 & - & $38(100 \%)$ & - & $71 \%(27)$ & $24 \%(9)$ & $5 \%(2)$ & $\mathrm{TTE}^{\ddagger}$ & Coronary artery disease & 0.03 \\
\hline Khosravi et al. ${ }^{28)}$ & $85^{*}$ & - & - & - & $57.6 \%(49)$ & - & - & $\begin{array}{l}\text { TTE (1 week) } \\
P I S A / E R O A\end{array}$ & $\dagger+$ & - \\
\hline Sehovic et al. ${ }^{29)}$ & 45 & - & $45(100 \%)$ & - & $53.3 \%(24)$ & $46.7 \%(21)$ & $0 \%$ & $\begin{array}{c}\text { TTE ( } 3 \text { months) } \\
\text { PISA }\end{array}$ & $\dagger$ & - \\
\hline Schubert et al. ${ }^{13)}$ & 423 & - & $407(96 \%)$ & $\begin{array}{c}\text { Rheumatic: } 12 \\
\text { Leaflet prolapse: } 4\end{array}$ & $44 \%(186)$ & $45 \%(189)$ & $11 \%(48)$ & $\begin{array}{l}\text { TTE/TOE (4 days) } \\
\text { PISA/EROA }\end{array}$ & $\dagger$ & - \\
\hline Wang et al. ${ }^{9)}$ & 49 & - & $49(100 \%)$ & - & - & - & - & $\begin{array}{c}\text { TTE + TOE (19 days) } \\
\text { PISA }\end{array}$ & - & - \\
\hline
\end{tabular}

\section{- Data not reported or analysed}

* Specific patients undergoing isolated AVR vs AVR + CABG not defined

¥ Patients with functional MR only

₹ Follow-up TTE/TOE timeframe not specified

${ }^{\dagger}$ No significant factors identified

MR: mitral regurgitation; TTE: transthoracic echocardiography; TOE: transoesophageal echocardiography; AVR: aortic valve replacement; CABG: coronary artery bypass graft; Postop: postoperative; PISA: proximal isovelocity surface area; EROA: effective regurgitant orifice area 
improvement in approximately half of patients, the reported percentages even in those studies that only included patients with secondary functional MR varied widely from $17.2 \%$ up to $72 \% .{ }^{16)}$ Notably in those studies that included patients with functional MR only and who had undertaken longer term echocardiographic follow-up of greater than 12 months, the range of improvement was $45 \%$ to $72 \%$, which may reflect the reverse LV remodeling over time. ${ }^{1,14,17,18)}$

The current American Heart Association/American College of Cardiology (AHA/ACC) guidelines for the management of valvular heart disease recommend mitral valve surgery, either repair or replacement, as a reasonable option for patients with chronic severe secondary MR who are undergoing AVR or CABG, or as an isolated procedure for patients with advanced New York Heart Association functional class. ${ }^{6,7,25)}$ In the context of moderate severity MR, these guidelines also propose that concomitant mitral valve repair is reasonable in patients with chronic moderate primary MR and that concomitant mitral valve repair may be considered in patients with chronic moderate secondary MR. ${ }^{6,7,25)}$ These recommendations acknowledge the progressive nature of MR and the potential utility of intervention for moderate severity MR to mitigate the future risk of progression. The 2017 European society of Cardiology/ European Association for Cardio-Thoracic Surgery (ESC/EACTS) guidelines recognize that chronic secondary MR is associated with worsening prognosis and outline a similar consensus for surgical intervention in such cases. ${ }^{24)}$

The guidelines acknowledge the challenging decision for clinicians and patients to either repair or replace in the context of moderate to severe MR. The AHA/ACC guidelines suggest that repair is still preferential to replacement, proposing that mitral valve replacement (MVR) may hold more surgical risk than repair. ${ }^{7,25)}$ However in the most recent 2020 AHA/ACC guidelines update, in profoundly symptomatic patients with severe secondary MR, the guidelines outline that chordal sparing MV replacement over MV repair may be reasonable. $\left.{ }^{6}\right)$ In 2016, Goldstein et al. reported an RCT of 251 patients undergoing either mitral valve repair or choral sparing replacement for chronic, severe ischaemic MR. ${ }^{26)}$ This multi-center study observed no significant difference between groups in left ventricular modeling or overall survival at 2-year follow-up; however, it did identify more frequent heart failure-related adverse events and hospital admissions in the mitral valve repair group. ${ }^{26)}$
The evaluation of MR is challenging. Mitral regurgitant flow is a complex and dynamic 3-dimensional process making 2-dimensional echocardiographic assessment difficult. The development of robust guidelines and consistent reporting of echocardiographic parameters used to assess MR severity is fundamental for the management of functional MR. Currently, a range of qualitative, semi-quantitative and quantitative parameters is still used and the criteria to assess severity can vary widely. ${ }^{7,23)}$

Color flow Doppler is the most common qualitative method of assessing MR severity. ${ }^{23)}$ However the size and extent of the regurgitant jet into the left atrium are inconsistent and do not linearly correlate with MR severity. The relative size and extent of MR Doppler flow can be influenced by left atrial size and pressure, chronicity of regurgitation, as well as hemodynamic factors. ${ }^{23,24)}$ Vena contracta width is also used as a semi-quantitative assessment of MR severity by measuring the cross-sectional width of the regurgitant jet. ${ }^{7,23)}$ However, the accuracy with which it reflects the true size and shape of the regurgitant orifice can be imprecise and operator dependent. ${ }^{23)}$

The flow convergence method is currently the most accurate quantitative method of evaluating MR severity. ${ }^{23,27)}$ By calculating the proximal isovelocity surface area (PISA) of a regurgitant colour flow jet, the effective regurgitant orifice area (EROA) of a mitral valve lesion to be accurately determined. ${ }^{23,27)}$ Most importantly, this method provides greater insight into the hemodynamic effects of MR severity on the LV and left atrium. ${ }^{27)}$ Using the PISA method, primary MR is classified into mild (EROA of $<20 \mathrm{~mm}^{2}$ and regurgitant volume of $<30 \mathrm{~mL}$ ), mild to moderate (EROA of $20 \mathrm{~mm}^{2}$ to $29 \mathrm{~mm}^{2}$ and regurgitant volume between $30 \mathrm{~mL}$ to $39 \mathrm{~mL}$ ), moderate to severe (EROA of $30 \mathrm{~mm}^{2}$ to $39 \mathrm{~mm}^{2}$ and regurgitant volume between $40 \mathrm{~mL}$ to $49 \mathrm{~mL}$ ) and severe (EROA of $\geq 40 \mathrm{~mm}^{2}$ and regurgitant volume $\geq 60 \mathrm{~mL}$ ). ${ }^{23)}$ Secondary functional $\mathrm{MR}$, on the other hand, is defined as an EROA $<20 \mathrm{~mm}^{2}$ and regurgitant volume $>30 \mathrm{~mL}$. $^{23)}$ The PISA method is highly recommended by the ESC/EACTS guidelines to determine MR severity for both central and eccentric lesions; however, it is not without its limitations. ${ }^{23,25,27)}$

This review demonstrates the variability in echocardiographic reporting standards for the assessment of MR severity over the last two decades. A total of 7 studies adopted the ESC/EACTS guideline-recommended flow convergence method, whilst the remaining 10 studies either utilized a variety of semi-quantitative or qualitative 
Cheung FP, et al.

measures or did not report the parameters used. Of these 7 studies, only 3 reported the absolute values used for EROA and regurgitant volume, which reflected the ESC/ EACTS guideline parameters. The increasing implementation of universal echocardiographic assessment guidelines will undoubtedly help the heart team decision-making process in the future. ${ }^{23)}$ Postoperative echocardiographic assessment should also involve longterm follow-up. In this review, the timeframe for postoperative echocardiography ranged from 1 week up to 36 months following AVR. Nine studies conducted transthoracic echocardiograms from 3 up to 36 months and 5 studies relied on echocardiographic evaluation at less than 1 month, which makes it unable to examine the effect of ventricular remodeling on MR improvement in the long term. ${ }^{24)}$ The afterload reduction and easing of left ventricular systolic pressure following AVR may provide an initial reduction in MR severity, but the progressive ventricular reverse remodeling in the long term remains the true determinant of sustained improvements in MR. ${ }^{10)}$ There are several preoperative parameters that have been associated with improvements in MR including LV end-diastolic volume, left ventricular mass, left atrial diameter and ejection fraction; however, these have been inconsistently reported in the current literature..$^{2,10,20,21)}$ The identification of more robust preoperative echocardiographic parameters requires consistent reporting of quantitative assessment methods used and this will ultimately help predict which patients exhibit an improvement in MR following an AVR. ${ }^{23)}$

The authors acknowledge the limitations of this review. The majority of studies included are retrospective, with only 3 prospective studies included. The surgical cohorts differed between studies with inclusion of primary and secondary MR etiology, whilst the echocardiographic assessment timeframes and reporting of MR severity also varied widely. The collective evidence presented in this review represents a heterogeneous pool of data that does not guide, with any certainty, when to address an incompetent mitral valve at the time of surgical AVR.

\section{Conclusion}

The decision for mitral valve intervention in patients presenting with intermediate degrees of concomitant functional MR and severe AS is undoubtedly challenging. The current literature does demonstrate that a substantial proportion of patients will exhibit an improvement in moderate functional MR severity following isolated surgical AVR with long-term follow-up of 12 months or more, but whether this improvement confers any mortality benefit remains unclear. In addition, those patients exhibiting persistent or worsening MR may be at an increased risk of hospital readmissions for heart failure and double valve intervention may therefore be considered in judiciously selected patients. It is clear that more robust randomized-controlled data, which incorporates a standardized set of echocardiographic parameters, are needed to help the interdisciplinary heart team identify which patients will benefit from isolated AVR or double valve intervention.

\section{Disclosure Statement}

There are no conflicts of interest to declare.

\section{Appendix 1 Medline (OVID: 1946-present) 15 July 2020}

1. Aortic valve replacement.mp or exp Heart Valve Replacement/

2. Mitral Valve Insufficiency/

3. Mitral regurgitation.mp

4. Mitral insuffiency.mp

5. 2 OR 3 OR 4

6. 1 AND 5

\section{Appendix 2 Inclusion criteria for studies}

1. Studies published after 2000 .

2. Age $>18$ years.

3. Surgical AVR for severe AS with concomitant MR.

4. Assessing change in severity of MR.

5. Postoperative TTE used to assess change in MR severity.

\section{References}

1) Moazami N, Diodato MD, Moon MR, et al. Does functional mitral regurgitation improve with isolated aortic valve replacement? J Card Surg 2004; 19: 444-8.

2) Wan CK, Suri RM, Li Z, et al. Management of moderate functional mitral regurgitation at the time of aortic valve replacement: is concomitant mitral valve repair necessary? J Thorac Cardiovasc Surg 2009; 137: 63540.e1.

3) Harling L, Saso S, Jarral OA, et al. Aortic valve replacement for aortic stenosis in patients with concomitant mitral regurgitation: should the mitral valve be dealt with? Eur J Cardiothorac Surg 2011; 40: 1087-96. 
4) Opie LH, Commerford PJ, Gersh BJ, et al. Controversies in ventricular remodelling. Lancet 2006; 367: 356-67.

5) Lansac E, Lim KH, Shomura Y, et al. Dynamic balance of the aortomitral junction. J Thorac Cardiovasc Surg 2002; 123: 911-8.

6) Bonow RO, O'Gara PT, Adams DH, et al. focused update of the 2017 ACC expert consensus decision pathway on the management of mitral regurgitation: a report of the American College of Cardiology Solution Set Oversight Committee. J Am Coll Cardiol 2020; 75: 2236-70.

7) Nishimura RA, Otto CM, Bonow RO, et al. AHA/ACC focused update of the 2014 AHA/ACC guideline for the management of patients with valvular heart disease: a report of the American College of Cardiology/American Heart Association Task Force on Clinical Practice Guidelines. J Am Coll Cardiol 2017; 70: 252-89.

8) Nishimura RA, Otto CM, Bonow RO, et al. AHA/ ACC guideline for the management of patients with valvular heart disease: executive summary: a report of the American College of Cardiology/American Heart Association Task Force on Practice Guidelines. J Am Coll Cardiol 2014; 63: 2438-88.

9) Wang W, Wang T, Piao H, et al. Change in functional moderate mitral regurgitation after aortic valve replacement. Braz J Cardiovasc Surg 2019; 34: 659-66.

10) Unger P, Plein D, Van Camp G, et al. Effects of valve replacement for aortic stenosis on mitral regurgitation. Am J Cardiol 2008; 102: 1378-82.

11) Vanden Eynden F, Bouchard D, El-Hamamsy I, et al. Effect of aortic valve replacement for aortic stenosis on severity of mitral regurgitation. Ann Thorac Surg 2007; 83: 1279-84.

12) Wyler S, Emmert MY, Biaggi P, et al. What happens to functional mitral regurgitation after aortic valve replacement for aortic stenosis? Heart Surg Forum 2013; 16: E238-42.

13) Schubert SA, Yarboro LT, Madala S, et al. Natural history of coexistent mitral regurgitation after aortic valve replacement. J Thorac Cardiovasc Surg 2016; 151: 1032-9, 1042.e1.

14) Joo HC, Chang BC, Cho $\mathrm{SH}$, et al. Fate of functional mitral regurgitation and predictors of persistent mitral regurgitation after isolated aortic valve replacement. Ann Thorac Surg 2011; 92: 82-7.

15) Takeda K, Matsumiya G, Sakaguchi T, et al. Impact of untreated mild-to-moderate mitral regurgitation at the time of isolated aortic valve replacement on late adverse outcomes. Eur J Cardiothorac Surg 2010; 37: 1033-8.

16) Christenson JT, Jordan B, Bloch A, et al. Should a regurgitant mitral valve be replaced simulataneously with a stenotic aortic valve? Tex Heart Inst J 2000; 27: 350-5.

17) Absil B, Dagenais F, Mathieu P, et al. Does moderate mitral regurgitation impact early or mid-term clinical outcome in patients undergoing isolated aortic valve replacement for aortic stenosis? Eur J Cardiothorac Surg 2003; 24: 217-22; discussion 22.

18) Goland S, Loutaty G, Arditi A, et al. Improvement in mitral regurgitation after aortic valve replacement. Isr Med Assoc J 2003; 5: 12-4.

19) Brasch AV, Khan SS, DeRobertis MA, et al. Change in mitral regurgitation severity after aortic valve replacement for aortic stenosis. Am J Cardiol 2000; 85: $1271-4$.

20) Tassan-Mangina S, Metz D, Nazeyllas P, et al. Factors determining early improvement in mitral regurgitation after aortic valve replacement for aortic valve stenosis: a transthoracic and transesophageal prospective study. Clin Cardiol 2003; 26: 127-31.

21) Aljadayel HA, Alkanj $H$. Effect of aortic valve replacement, for aortic stenosis, on concomitant mitral valve regurgitation. Turkiye Klinikleri Cardiovasc Sci 2015; 27: 22-7.

22) Caballero-Borrego J, Gómez-Doblas JJ, CabreraBueno F, et al. Incidence, associated factors and evolution of non-severe functional mitral regurgitation in patients with severe aortic stenosis undergoing aortic valve replacement. Eur J Cardiothorac Surg 2008; 34: 62-6.

23) Lancellotti P, Tribouilloy C, Hagendorff A, et al. Recommendations for the echocardiographic assessment of native valvular regurgitation: an executive summary from the European Association of Cardiovascular Imaging. Eur Heart J Cardiovasc Imaging 2013; 14: 611-44.

24) Baumgartner H, Falk V, Bax JJ, et al. ESC/EACTS Guidelines for the management of valvular heart disease. Eur Heart J 2017; 38: 2739-91.

25) Nishimura RA, Otto CM, Bonow RO, et al. AHA/ACC guideline for the management of patients with valvular heart disease: a report of the American College of Cardiology/American Heart Association Task Force on Practice Guidelines. J Am Coll Cardiol 2014; 63: e57-185.

26) Goldstein D, Moskowitz AJ, Gelijns AC, et al. Twoyear outcomes of surgical treatment of severe ischemic mitral regurgitation. N Engl J Med 2016; 374: 344-53.

27) Enriquez-Sarano M, Miller FA, Hayes SN, et al. Effective mitral regurgitant orifice area: clinical use and pitfalls of the proximal isovelocity surface area method. J Am Coll Cardiol 1995; 25: 703-9.

28) Khosravi A, Sheykhloo H, Karbasi-Afshar R, et al. Echocardiographic changes after aortic valve replacement: Does the failure rate of mitral valve change? ARYA Atheroscler 2015; 11: 147-52.

29) Sehovic $S$, Talic A, Kacila $M$, et al. The influence of aortic valve replacement on functional moderate-tosevere mitral regurgitation in patients with aortic valve stenosis. Acta Inform Med 2015; 23: 147-50. 